\title{
An Aminopyrrolidinyl Phosphonates-A New Class of Antibiotics: Facile Synthesis and Predicted Biological Activity
}

\author{
Abed Al Aziz Al Quntar1,2*, Hasan Dweik², Ahmad Jabareen'1, Tatyana A. Gloriozova3 ${ }^{3}$ \\ Valery M. Dembitsky4 \\ ${ }^{1}$ Department of Material Engineering, Faculty of Engineering, Al-Quds University, Jerusalem, Palestine \\ ${ }^{2}$ Faculty of Chemistry and Chemical Technology, Al-Quds University, Jerusalem, Palestine \\ ${ }^{3}$ Institute of Biomedical Chemistry, Moscow, Russia \\ ${ }^{4}$ Centre for Applied Research and Innovation, and Entrepreneurship, Lethbridge College, Lethbridge, Canada \\ Email: *abedalaziz@staff.alquds.edu
}

How to cite this paper: Al Quntar, A.A.A. Dweik, H., Jabareen, A., Gloriozova, T.A. and Dembitsky, V.M. (2020) An Aminopyrrolidinyl Phosphonates-A New Class of Antibiotics: Facile Synthesis and Predicted Biological Activity. International Journal of Organic Chemistry, 10, 170-181. https://doi.org/10.4236/ijoc.2020.104013

Received: November 16, 2020 Accepted: December 26, 2020 Published: December 29, 2020

Copyright $\odot 2020$ by author(s) and Scientific Research Publishing Inc. This work is licensed under the Creative Commons Attribution International License (CC BY 4.0).

http://creativecommons.org/licenses/by/4.0/

\begin{abstract}
A novel class of aminopyrrolidinyl phosphonates was synthesized in $74 \%$ $80 \%$ isolated yield by the addition of three-fold excess of primary amines to diethyl 4-chloro-1-butynylphosphonates. The reaction was carried out at room temperature and in the absence of solvent or catalyst to give solely compounds which showed predicted biological activity based on PASS program. Some of the synthesized derivatives of antibiotics exhibit properties for the treatment of stroke, the treatment of acute neurological disorders, and can also be acetyl esterase inhibitors.
\end{abstract}

\section{Keywords}

Alkynylphosphonates, Cyclization, Pyrrolidine, $\beta$-Aminophosphonates, Amine Addition, Antibiotics

\section{Introduction}

Natural antibiotics are produced by many microorganisms and are of great interest in medicine and pharmacology [1] [2] [3] [4]. It is well known that some antibiotics, such as K-26, SF-2513, FR-33289, and SF-2312 contain a C-P (carbon-phosphorus) bond that belongs to the category of phosphonates [5] [6] [7] [8]. The targeted synthesis of phosphonates for pharmacological utility is also fairly well described in several reviews [9] [10] [11] [12] [13].

For instance, a pyrrolidinyl phosphonates class (known as antibiotic SF-2312) 
is a natural antibiotic which is produced by the actinomycete Micromonospora sp. It has not only showed activity as an enolase inhibitor but also found to be one of the most potent natural inhibitors of glycolysis which in turn inhibit cell proliferation [14] [15] [16] [17] [18]. Later, the synthesis of this antibiotic and its analogues has been described by various researchers [19] [20] [21].

In the same context, pharmacologists always agree that the biological activity of both natural and synthetic compounds is related to the chemical structure [22]. Therefore, there are a large number of available computer programs that can evaluate the probability of an organic compound activity to be a drug [23] [24]. For instance, PASS computer program contains a library with information of about 1 million chemical compounds and more than 10,000 biological activities [25]. An algorithm for the practical use of PASS has been described in detail in several publications [26] [27] [28].

Previously, few pyrrolidinylphosphonates and pyrrolidinylphosphonic acids (1-12, Figure 1) were synthesized for the purpose of testing their biological activity. Generally, they were obtained either from readily available pyrrolidine ring or by multistep cyclization reactions. But there is no one general direct method to produce them and a brief summary for their synthesis is described below:

Initially, the antibiotic SF-2312 (1) and its analogue (3) were synthesized by a multistep reaction sequence in which ethyl diethoxyphosphorylacetate was converted to N-benzyloxy-2-(diethoxyphosphoryl)-pent-4-enamide followed by oxidative cleavage and hydrolysis [21]. Later, studies showed that their antibiotic activity is due to the inhibition of a glycolytic enzyme called enolase [14] [15] [16]. The reaction of pentanedial with acetamide and acetyl chloride in the presence of phosphorylating agent afforded the pyrrolidinyldiphosphonic acid (2) [29]. The combination of the Kabachnik-Fields reaction with a subsequent ring closure of 5-chloro-2-pentanone with ammonia and diethyl phosphonate produced (4) [30].

The pyrrolidinylphosphonates (5-6) were prepared from readily available 2-methylpyrroline and diethyl phosphite [31]. The SF-2312 (1) antibiotic analogues (7-10) were obtained using 1-benzyloxy-3-bromopyrrolidin-2,5-dione through Michaelis-Arbusov reaction with trialkyl phosphites followed by alkylation. However, their biological activity was not determined [19] [20].

Asymmetric synthesis of $\beta$-amidophosphonate (11) was achieved by $\mathrm{Di}$ els-Alder reaction between the vinylphosphonate and chiral aminodiene [32]. Finally, three-component decarboxylative coupling of proline with aldehydes and dialkyl phosphite was used to afford the corresponding pyrrolidinylphosphonates (12) [33].

\section{Results and Discussion}

We recently reported the synthesis of $\mathrm{N}$-substituted pyrrolidinyl-methylphosphonate (14) by addition of amines to (Z)-diethyl (5-chloropent-1-en-1-yl)phosphonate 13 (Scheme 1) [34]. 

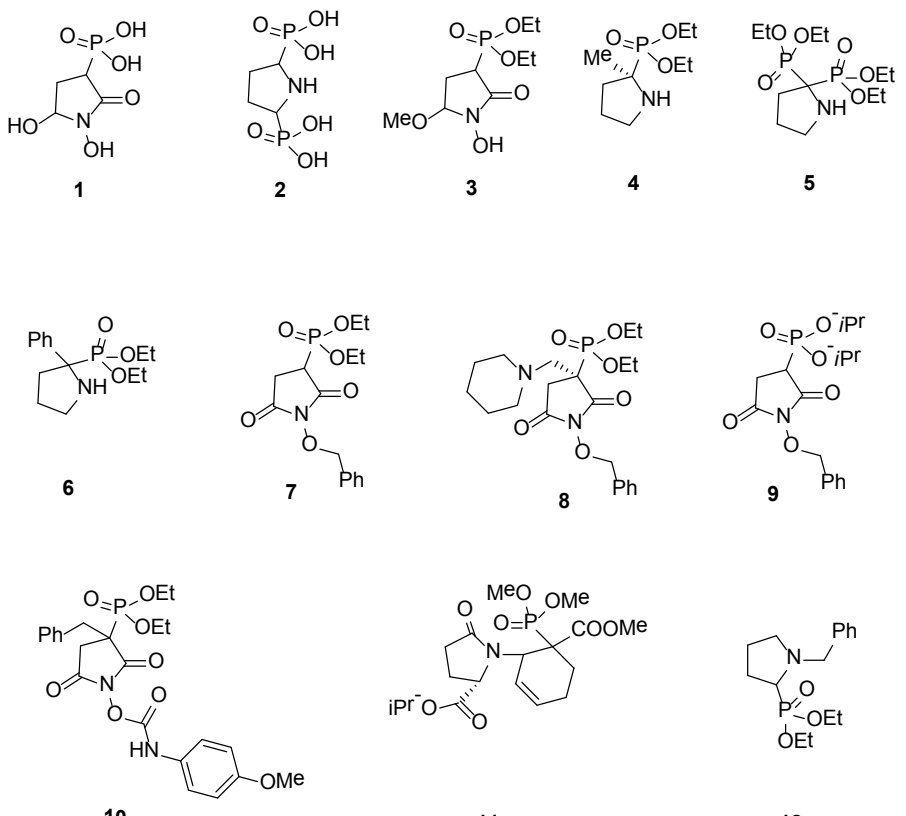

10

11

12

Figure 1. Pyrrolidinyl phosphonates 1-12.

$\mathrm{Cl}$

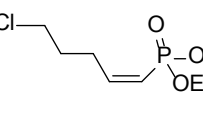

13

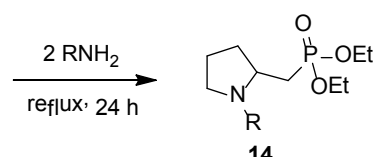

14

$\mathrm{R}=\mathrm{alkyl}, \mathrm{ar}_{\mathrm{yl}}$

Scheme 1. Formation of pyrrolidines N-substituted pyrrolidinyl-methylphosphonate 14 .

In addition, we investigated the reaction of 4-chloro-1-butentynylphosphonate 15 with an equivalent number of amines which gave

2-amino-cyclobutenylphosphonates [35]. Interestingly, a different novel class of compounds (17a-h) was obtained by tuning of the reaction conditions.

Being encouraged by these results, we determined to study amine addition on a shorter chain alkynylphosphonate that has not been explored before. Accordingly, we prepared 4-chloro-1-butentynylphosphonate 15 in our lab by substituting the hydroxy group in but-3-yne-1-ol using thionyl chloride under reflux. After isolation of the product by distillation, it was lithiated using $n-\mathrm{BuLi}$, and was reacted with chlorophosphonates.

Herein, we report a very facile method for the synthesis of novel aminopyrrolidinyl phosphonates (17a-h) and their predicted biological activity using the PASS program.

Thus, when three equivalents of $i$-propylamine were added to (15), the pyrrolidine structure diethyl (1-isopropyl-3-(isopropylamino)pyrrolidin-2-yl)phosphonate (17a) was gained in $80 \%$ yield (Scheme 2 ). Similarly, products (17b-h) were produced in $(74 \%-79 \%)$ isolated yield by treatment of 15 with a different primary amine. 


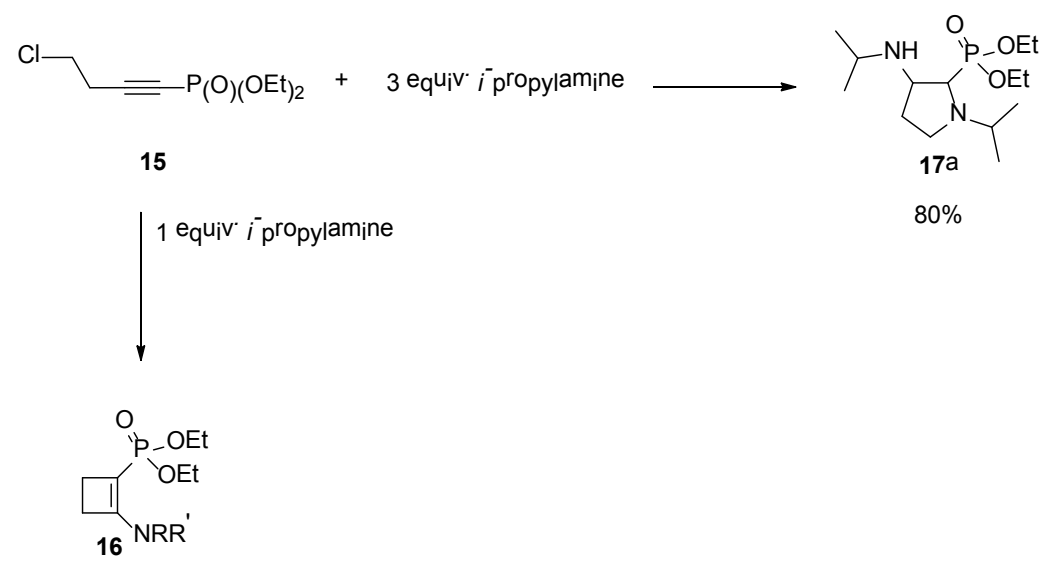

Scheme 2. Formation of pyrrolidinylphosphonate 17a.

The reaction was carried out at $25^{\circ} \mathrm{C}$ for $12 \mathrm{~h}$. and the products 17 were isolated by silica gel column chromatography in good yields $(74 \%-80 \%)$ and were characterized by NMR, GC/MS, and by elemental analysis. The multiplets in the regions $\sim(1.3-2.0 \mathrm{ppm}), \sim(2.1-3.0 \mathrm{ppm})$ in the ${ }^{1} \mathrm{H}$ NMR spectrum, precisely the doublet of doublet in the region $\sim 2 \mathrm{ppm}$ that corresponds to the hydrogen on $\mathrm{C} 1$ split by phosphorus and the hydrogen on $\mathrm{C} 2$ which resonated as a multiplet at $\sim 3 \mathrm{ppm}$, together with the carbons in the regions $\sim(30,32,45,55 \mathrm{ppm})$ in the ${ }^{13} \mathrm{C}$ NMR spectra are indicative of the pyrrolidine ring. Besides, the ${ }^{31} \mathrm{P}$ NMR that resonated chemical shifts at $\sim 31 \mathrm{ppm}$, the GC/MS and the elemental analysis are all evidence of structure 17 . These results were also supported by 2-D Cosy and ${ }^{1} \mathrm{H}^{13} \mathrm{C}$ HSQCSI NMR of $17 \mathrm{~d}$ in which a high correlation was observed.

As described above, pyrrolidines 1-12 are of predicted biologically active compounds despite the multistep procedures for their preparation.

This process represents a general one-pot method for the synthesis of novel oily amino-pyrrolidinyl phosphonates (17a-h) which have not been reported before. In addition, they are thermally and air-stable compounds at room temperature, and are soluble in most organic solvents. Besides, this cyclization reaction is general for both aliphatic and aromatic primary amines as shown in Table 1. In addition, they are of potent biological activity precisely as antibiotics analogous to compounds 1-12 as shown in Table 2.

Unlike primary amines, when secondary amines were used, no heterocycles were detected and only 2 -amino-cyclobutenylphosphonates 16 were obtained.

A suggested mechanism for this reaction can be attributed to initial addition of the amine on the carbon-carbon double bond to give a zwitterionic intermediate followed by proton transfer. Then, another hydroamination reaction took place on the double bond in the presence of excess amine to give the intermediate (20). After, nucleophilic attack of the nitrogen atom on $\mathrm{C} 1$ onto the carbon $\mathrm{C}-\mathrm{Cl}$ by $\mathrm{S}_{\mathrm{N}} 2$ fashion, an aminopyrrolidinyl phosphonate (17) was produced (Scheme 3) [31] [32] [33] [34]. 
Table 1. Synthesis of pyrrolidinylphosphonates 17a-h.

17

\begin{tabular}{ccccc}
\hline Entry & Products & Amine (3.1 equiv.) & $\mathbf{R}$ & ${\text { Isolated Yield }{ }^{\mathrm{a}} \text {, \% (reaction conversion) }}^{\mathbf{b}}$ \\
\hline 1 & $17 \mathrm{a}$ & $i$-propylamine & $i$-propyl & $80(>98)$ \\
2 & $17 \mathrm{~b}$ & $t$-butylamine & $t$-butyl & $74(>98)$ \\
3 & $17 \mathrm{c}$ & benzylamine & benzyl & $78(>98)$ \\
4 & $17 \mathrm{~d}$ & amylamine & $n$-pentyl & $75(>98)$ \\
5 & $17 \mathrm{e}$ & $n$-butylamine & phenyl & $76(>98)$ \\
6 & $17 \mathrm{f}$ & phenylamine & $n$-heptyl & $75(>98)$ \\
7 & $17 \mathrm{~g}$ & n-heptylamine & phenylethyl & $79(>98)$ \\
\hline
\end{tabular}

${ }^{\mathrm{a}}$ After silica gel chromatography; ${ }^{\mathrm{b}}>98 \%$ as determined by GC/MS and ${ }^{31} \mathrm{P}$ NMR.

Table 2. Predicted biological activity of compounds 1-17.

\begin{tabular}{|c|c|c|c|}
\hline No. & $\begin{array}{l}\text { Experimental } \\
\text { reported Activity, } \\
(\mathrm{Pa})^{\star}\end{array}$ & Predicted theoretical biological activity, $(\mathrm{Pa})^{*}$ & $\begin{array}{l}\text { Related } \\
\text { reference }\end{array}$ \\
\hline 1 & $\begin{array}{l}\text { Inhibitor of the } \\
\text { glycolytic enzyme } \\
\text { enolase } 2\end{array}$ & $\begin{array}{l}\text { Stroke treatment (0.761); Neuroprotector }(0.744) \text {; Acute neurologic disorders treatment }(0.663) \text {; } \\
\text { Antineoplastic (sarcoma) (0.646); Antinephrotoxic (0.587); Antiseborrheic }(0.578)\end{array}$ & $(15,16,21)$ \\
\hline 2 & Not reported & $\begin{array}{l}\text { Stroke treatment (0.979); Antiviral (0.897); Antiviral (HIV) }(0.887) \\
\text { Antinephrotoxic (0.701); Bone formation stimulant }(0.653)\end{array}$ & $(29,31)$ \\
\hline 3 & Not reported & Antibiotic Glycopeptide-like (0.984); Stroke treatment (0.538) Antischistosomal (0.510) & (21) \\
\hline 4 & Not reported & $\begin{array}{l}\text { Antiarthritic }(0.963) \text {; Anti-inflammatory }(0.942) \text {; Acute neurologic disorders treatment }(0.765) \text {; } \\
\text { Bone formation stimulant }(0.615) \text { Antinephrotoxic }(0.608) \text {; Stroke treatment }(0.567) \\
\text { Leukopoiesis stimulant }(0.542) \text {; Bone diseases treatment }(0.539)\end{array}$ & (30) \\
\hline 5 & Not reported & $\begin{array}{l}\text { Bone formation stimulant }(0.813) \text {; Bone diseases treatment }(0.803) \text { Acute neurologic disorders } \\
\text { treatment }(0.650) \text {; Antinephrotoxic }(0.604) \text { Antiosteoporotic }(0.560) \text {; Leukopoiesis stimulant }(0.526)\end{array}$ & $(31)$ \\
\hline 6 & Not reported & $\begin{array}{l}\text { Acute neurologic disorders treatment }(0.834) \text {; Stroke treatment }(0.583) \text { Bone formation stimulant } \\
(0.574) \text {; Bone diseases treatment }(0.536) \text { Anesthetic general }(0.517) \text {; Leukopoiesis stimulant }(0.506)\end{array}$ & $(31)$ \\
\hline 7 & Not reported & Acetylesterase inhibitor (0.618); Anticonvulsant (0.533) & $(19,20)$ \\
\hline 8 & Not reported & Anticonvulsant (0.664); Acetylesterase inhibitor (0.637); Acute neurologic disorders treatment (0.513) & $(19,20)$ \\
\hline 9 & Not reported & Acetylesterase inhibitor (0.687); Myasthenia Gravis treatment (0.562) & $(19,20)$ \\
\hline 10 & Not reported & Cognition disorders treatment $(0.765)$ & $(19,20)$ \\
\hline 11 & Not reported & Calcium regulator (0.548); Antihypertensive (0.503) & $(32)$ \\
\hline 12 & Not reported & $\begin{array}{l}\text { Acute neurologic disorders treatment }(0.852) \text {; Stroke treatment }(0.582) \text { Bone diseases treatment } \\
(0.580) \text {; Bone formation stimulant }(0.531)\end{array}$ & $(33)$ \\
\hline $17 \mathrm{a}$ & Not reported & Acetylesterase inhibitor (0.611); Insulinotropin antagonist (0.568) & Present study \\
\hline $17 \mathrm{~b}$ & Not reported & Spasmolytic, urinary $(0.954)$ & Present study \\
\hline $17 c$ & Not reported & Acetylesterase inhibitor (0.572); Immunostimulant (0.522) & Present study \\
\hline $17 \mathrm{~d}$ & Not reported & Acetylesterase inhibitor (0.618); Immunostimulant (0.544) & Present study \\
\hline $17 \mathrm{e}$ & Not reported & Acetylesterase inhibitor (0.618); Immunostimulant (0.544) & Present study \\
\hline $17 f$ & Not reported & Purinergic P2Y2 antagonist (0.512) & Present study \\
\hline $17 \mathrm{~g}$ & Not reported & No activities with $\mathrm{Pa}>0.5$ & Present study \\
\hline $17 \mathrm{~h}$ & Not reported & General pump inhibitor $(0.600)$ & Present study \\
\hline
\end{tabular}

*Only activities with $\mathrm{Pa}>0.5$ are shown. 

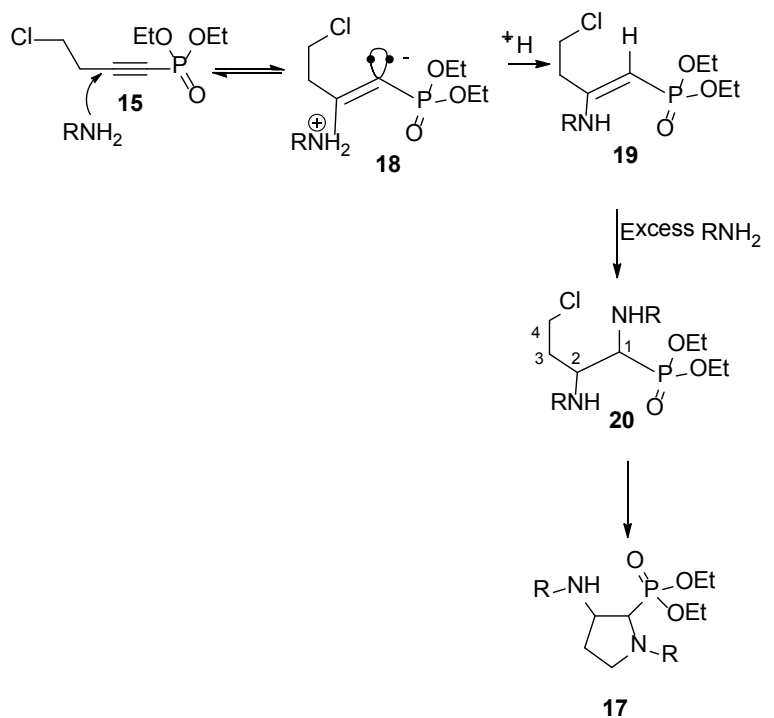

Scheme 3. Suggested mechanism for the synthesis of the compounds 17.

This suggested mechanism is also supported by the fact that when secondary amines were used, no hetero cycles were detected and only

2 -amino-cyclobutenylphosphonates 16 were obtained. In addition, this reaction was restricted to 4-chloro-1-butynylphosphonate 15 . When longer chloro alkynylphosphonates chains were used no heterocyclic structure was observed apparently due to the proximity of the chloro substituted carbon to the conjugated system of the triple bond and the phosphonate group. After the synthesis of compounds (17a-h), their biological activity was screened utilizing the PASS program and were found to be of predicted pharmacological usefulness as listed in Table 2.

\section{Conclusion}

In conclusion, a novel aminopyrrolidinyl phosphonate class of compounds (17a-h) was smoothly obtained by addition of primary amines to

4-chloro-1-butynylphosphonate in the absence of solvent or catalyst and relatively in a satisfactory isolated yield. In addition, manifestations of biological activities of the above compounds were observed using PASS program. This reaction can be of interest for the related chemist's community to apply this reaction to other functional groups instead of phosphonates and for the biologists to test their predicted biological activity.

\section{Experimental}

The ${ }^{1} \mathrm{H},{ }^{13} \mathrm{C}$, and ${ }^{31} \mathrm{P}$ NMR spectra were recorded from solutions in $\mathrm{CDCl}_{3}$ on a Varian Mercury 300 spectrometer at 300, 75.5, and $121.4 \mathrm{MHz}$, respectively; the chemical shifts were measured relative to TMS $\left({ }^{1} \mathrm{H},{ }^{13} \mathrm{C}\right)$ and $\mathrm{H}_{3} \mathrm{PO}_{4}$. The mass spectra (EI) were recorded on an HP G1800A GCD GC/MS instrument using a 30-m methyl silicone column. 


\section{Synthesis of Diethyl (4-chlorobut-1-yn-1-yl)phosphonate (15)}

Since the starting material 4-chlorobut-1-yne was not commercially available as usual, it was prepared in our lab [35].

Synthesis of aminopyrrolidinyl phosphonates (17a-h)

Typical procedure for the synthesis of diethyl (1-isopropyl-3-(isopropylamino)pyrrolidin-2-yl)phosphonate 17a.

To diethyl (4-chlorobut-1-yn-1-yl)phosphonate $(0.22 \mathrm{~g}, 1 \mathrm{mmol})$ was added $(0.23 \mathrm{~g}, 3.5 \mathrm{mmol})$ of isopropylamine in a $10 \mathrm{~mL}$ round bottom flask. After stirring at $25^{\circ} \mathrm{C}$ for $12 \mathrm{~h}$. the reaction mixture was washed with $0.1 \mathrm{~N} \mathrm{NaOH}$ solution and the product was extracted with $\left(2 \times 20 \mathrm{~mL} \mathrm{CH} \mathrm{Cl}_{2}\right)$, dried over $\mathrm{MgSO}_{4}$, concentrated using a rotary evaporator and the oily product was separated on a silica gel column and was obtained in $80 \%$ isolated yield (10\% methanol:90\% dichloromethane), which was then analyzed by GC/MS, elemental analysis, and NMR spectroscopy.

${ }^{1} \mathrm{H}$ NMR (300 MHz, Chloroform d): $\delta 1.01\left(\mathrm{~d}, 6 \mathrm{H}, J_{\mathrm{HH}}=6.3 \mathrm{~Hz}\right), 1.11(\mathrm{~d}, 6 \mathrm{H}$, $\left.J_{\mathrm{HH}}=6.3 \mathrm{~Hz}\right), 1.30\left(\mathrm{t}, 6 \mathrm{H}, J_{\mathrm{HH}}=6.9 \mathrm{~Hz}\right), 1.65-2.30$ (overlap, $\left.3 \mathrm{H}\right), 2.65-70(\mathrm{~m}$, $1 \mathrm{H}), 2.78-2.88$ (overlap, $2 \mathrm{H}), 3.02-3.15(\mathrm{~m}, 1 \mathrm{H}), 3.42-3.48(\mathrm{~m}, 1 \mathrm{H}), 4.03$ 4.12 (m, 4H); ${ }^{31} \mathrm{P}$ NMR (121.4 MHZ, Chloroform d): $\delta 31.38 ;{ }^{13} \mathrm{C}$ NMR (75.5 $\mathrm{MHz}$, Chloroform d): $15.8\left(\mathrm{~d},{ }^{3} J_{\mathrm{PC}}=6.8\right), 15.8,15.9,32.0\left(\mathrm{~d},{ }^{1} J_{\mathrm{PC}}=126.7 \mathrm{~Hz}\right)$, 44.4, $50.8\left(\mathrm{~d},{ }^{2} J_{\mathrm{PC}}=3.2 \mathrm{~Hz}\right), 53.1,60.4\left(\mathrm{~d},{ }^{2} J_{\mathrm{PC}}=8.6 \mathrm{~Hz}\right), 62.1,63.2 ; \mathrm{MS}(\mathrm{EI}): \mathrm{m} / \mathrm{z}$ (\%) 306 (10.5), 305 (15.1), 287 (20.0), 235 (22.3), 168 (35.0), 152 (18.8), 123 (30.0), 110 (41.3), 84 (100), 70 (56.7), 42 (19.9); Anal. Calcd. for $\mathrm{C}_{14} \mathrm{H}_{31} \mathrm{~N}_{2} \mathrm{O}_{3} \mathrm{P}: \mathrm{C}$, 54.88; H, 10.20; N, 9.14; P, 10.11. Found: C, 55.09; H, 10.37; N, 8.95; P, 9.97.

\section{Synthesis of diethyl}

\section{(1-(tert-butyl)-3-(tert-butylamino)pyrrolidin-2-yl)phosphonate 17b.}

Identical to procedure $17 \mathrm{a}$ except adding t-butylamine and was obtained in $74 \%$ isolated yield.

${ }^{1} \mathrm{H}$ NMR (300 MHz, Chloroform d): $\delta 1.09$ (s, 9H), $1.11(\mathrm{~s}, 9 \mathrm{H}), 1.31(\mathrm{dt}, 6 \mathrm{H}$, $J_{\mathrm{HH}}=7.2 \mathrm{~Hz},{ }^{3} J_{\mathrm{HP}}=2.1 \mathrm{~Hz}$ ), $1.63-2.22$ (overlap, $3 \mathrm{H}$ ), $2.61-2.67$ (overlap m, 2H), 3.21 - $3.45(\mathrm{~m}, 1 \mathrm{H}), 4.06-4.13(\mathrm{~m}, 4 \mathrm{H}) ;{ }^{31} \mathrm{P}$ NMR (121.4 MHZ, Chloroform d): $\delta 30.38 ;{ }^{13} \mathrm{C}$ NMR (75.5 MHz, Chloroform d): 16.4 (d, ${ }^{3} J_{\mathrm{PC}}=6.3$ ), 28.8, 30.0, $34.5\left(\mathrm{~d},{ }^{1} \mathrm{JPC}_{\mathrm{PC}}=132.4 \mathrm{~Hz}\right), 39.7,47.0,50.6,50.8\left(\mathrm{~d},{ }^{2} J_{\mathrm{PC}}=3.0 \mathrm{~Hz}\right), 60.4\left(\mathrm{~d},{ }^{2} J_{\mathrm{PC}}=8.6\right.$ Hz); MS(EI): m/z (\%) 334 (0.70), 319 (2.5), 289 (7.8), 277 (17.8), 244 (27.8), 220 (70.5), 186 (51.1), 136 (100), 89 (55.1), 57 (67.8); Anal. Calcd. for $\mathrm{C}_{16} \mathrm{H}_{35} \mathrm{~N}_{2} \mathrm{O}_{3} \mathrm{P}$ : C, 57.46; H, 10.55; N, 8.38; P, 9.26. Found: C, 58.18; H, 10.73; N, 8.21; P, 9.12.

\section{Synthesis of}

\section{diethyl(1-benzyl-3-(benzylamino)pyrrolidin-2-yl)phosphonate 17c.}

Identical to procedure 17a except adding benzylamine and was obtained in $78 \%$ isolated yield.

${ }^{1} \mathrm{H}$ NMR (300 MHz, Chloroform d): ${ }^{1} \mathrm{H}$ NMR (300 MHz, Chloroform d): $\delta$ $1.26\left(\mathrm{dt}, 6 \mathrm{H}, J_{\mathrm{HH}}=6.9 \mathrm{~Hz},{ }^{3} J_{\mathrm{HP}}=1.51 \mathrm{~Hz}\right.$ ), $1.69-2.18$ (overlap, 3H), $2.64-283$ (m, 2H), 3.05 - $3.14(\mathrm{~m}, 1 \mathrm{H}), 3.76(\mathrm{~s}, 2 \mathrm{H}), 3.77(\mathrm{~s}, 2 \mathrm{H}), 4.00$ - $4.11(\mathrm{~m}, 4 \mathrm{H}), 7.21$ 7.33 (overlap, 10); ${ }^{31} \mathrm{P}$ NMR (121.4 MHZ, Chloroform d): $\delta 30.98 ;{ }^{13} \mathrm{C}$ NMR (75.5 
$\mathrm{MHz}$, Chloroform d): $16.1\left(\mathrm{~d},{ }^{3} J_{\mathrm{PC}}=7.4\right), 30.5,40.8,47.5\left(\mathrm{~d},{ }^{1} J_{\mathrm{PC}}=126.7 \mathrm{~Hz}\right)$, $\left.45.5,55.0,60.2\left(\mathrm{~d},{ }^{2} J_{\mathrm{PC}}=4.0 \mathrm{~Hz}\right), 60.9\left(\mathrm{~d},{ }^{2}\right)_{\mathrm{PC}}=5.7 \mathrm{~Hz}\right), 127.7,127.0,128.2$, 141.5; MS(EI): $m / z$ (\%) 402 (3.1), 401 (4.7), 325 (15.4), 311(17.7), 295 (17.9), 266 (30.4), 218 (44.9), 186 (22.3), 136 (60.1), 104 (33.6), 91 (100), 77 (55.8), 65 (18.8), 54 (26.8); Anal. Calcd. for $\mathrm{C}_{22} \mathrm{H}_{31} \mathrm{~N}_{2} \mathrm{O}_{3} \mathrm{P}: \mathrm{C}, 65.65 ; \mathrm{H}, 7.76 ; \mathrm{N}, 6.96 ; \mathrm{P}, 7.70$. Found: C, 65.47; H, 7.64; N, 7.10; P, 7.81 .

\section{Synthesis of diethyl}

\section{(1-pentyl-3-(pentylamino)pyrrolidin-2-yl)phosphonate17d.}

Identical to procedure 17a except adding amylamine and was obtained in $75 \%$ isolated yield.

${ }^{1} \mathrm{H}$ NMR (300 MHz, Chloroform d): $\delta 0.89\left(\mathrm{t}, 3 \mathrm{H}, J_{\mathrm{HH}}=5.7 \mathrm{~Hz}\right), 0.92(\mathrm{t}, 3 \mathrm{H}$, $J_{\mathrm{HH}}=5.7 \mathrm{~Hz}$ ), $1.31\left(\mathrm{dt}, 6 \mathrm{H}, J_{\mathrm{HH}}=7.2 \mathrm{~Hz},{ }^{3} J_{\mathrm{HP}}=2.1 \mathrm{~Hz}\right.$ ), $1.21-1.34$ (overlap, $8 \mathrm{H}$ ), $1.40-1.51(\mathrm{~m}, 2 \mathrm{H}), 1.53-1.70(\mathrm{~m}, 2 \mathrm{H}), 1.80-2.21$ (overlap, $3 \mathrm{H}$ ), $2.55-2.80$ (overlap, 4H), $2.81-2.90((\mathrm{~m}, 1 \mathrm{H}), 3.00-3.05(\mathrm{~m}, 1 \mathrm{H}), 3.06-3.15(\mathrm{~m}, 1 \mathrm{H}), 4.06$ - 4.15 (m, 4H); ${ }^{31} \mathrm{P}$ NMR (121.4 MHZ, Chloroform d): $\delta 31.47 ;{ }^{13} \mathrm{C}$ NMR $(75.5$ $\mathrm{MHz}$, Chloroform d): 13.9, 14.1, 16.4 (d, ${ }^{3} \mathrm{P}_{\mathrm{PC}}=6.3$ ), 22.4, 22.6, 26.4, 27.9, 29.2, 29.5, 29.8, $30.1\left(\mathrm{~d},{ }^{1} \mathrm{JPC}_{\mathrm{PC}}=137.4 \mathrm{~Hz}\right), 32.3,46.5,47.1,48.9\left(\mathrm{~d},{ }^{2} J_{\mathrm{PC}}=3.0 \mathrm{~Hz}\right), 55.4$, $61.4\left(\mathrm{~d},{ }^{2} J_{\mathrm{PC}}=8.6 \mathrm{~Hz}\right)$; MS(EI): $\mathrm{m} / \mathrm{z}(\%) 362$ (0.8), 319 (0.9), 279 (1.1), 264 (8.4), 256 (2.0), 222 (15.0), 194 (42.9), 142 (28.1), 128 (100), 98 (28.8), 82 (14.3), 56 (34.4), 43 (49.8), 29 (64.2); Anal. Calcd. for $\mathrm{C}_{18} \mathrm{H}_{39} \mathrm{~N}_{2} \mathrm{O}_{3} \mathrm{P}: \mathrm{C}, 59.64 ; \mathrm{H}, 10.84 ; \mathrm{N}$, 7.73; P, 8.54. Found: C, 59.77; H, 10.97; N, 7.60; P, 8.41.

Synthesis of diethyl (1-butyl-3-(butylamino)pyrrolidin-2-yl)phosphonate 17 e.

Identical to procedure $17 \mathrm{a}$ except adding butylamine and was obtained in $77 \%$ isolated yield.

${ }^{1} \mathrm{H}$ NMR (300 MHz, Chloroform d): $\delta 0.88\left(\mathrm{t}, 3 \mathrm{H}, J_{\mathrm{HH}}=5.7 \mathrm{~Hz}\right), 0.96(\mathrm{t}, 3 \mathrm{H}$, $J_{\mathrm{HH}}=5.7 \mathrm{~Hz}$ ), $1.31\left(\mathrm{dt}, 6 \mathrm{H}, J_{\mathrm{HH}}=7.2 \mathrm{~Hz},{ }^{3} J_{\mathrm{HP}}=2.1 \mathrm{~Hz}\right.$ ), $1.35-1.48$ (overlap, $10 \mathrm{H}), 1.60-2.17$ (overlap, 7H), $2.90-3.10(\mathrm{~m}, 1 \mathrm{H}), 4.03-4.15(\mathrm{~m}, 4 \mathrm{H}) ;{ }^{31} \mathrm{P}$ NMR (121.4 MHZ, Chloroform d): $\delta 31.85 ;{ }^{13} \mathrm{C}$ NMR (75.5 MHz, Chloroform d): 13.6, 14.0, $16.2\left(\mathrm{~d},{ }^{3} J_{\mathrm{PC}}=6.5\right), 23.0,23.8,28.3,29.8,30.3,31.5\left(\mathrm{~d},{ }^{1} J_{\mathrm{PC}}=140.2\right.$ $\mathrm{Hz}), 33.5,48.1,49.3\left(\mathrm{~d},{ }^{2} J_{\mathrm{PC}}=3.2 \mathrm{~Hz}\right), 50.0,53.1,62.0\left(\mathrm{~d},{ }^{2} J_{\mathrm{PC}}=8.4 \mathrm{~Hz}\right)$; MS(EI): $m / z$ (\%) 334 (0.9), 333 (1.0), 319 (16.8), 304 (40.2), 289 (17.6), 276 (66.1), 219 (32.2), 189 (100), 135 (47.8), 89 (41.0), 57 (44.1); Anal. Calcd. for $\mathrm{C}_{16} \mathrm{H}_{35} \mathrm{~N}_{2} \mathrm{O}_{3} \mathrm{P}: \mathrm{C}, 57.46 ; \mathrm{H}, 10.55 ; \mathrm{N}, 8.38 ; \mathrm{P}, 9.26$. Found: $\mathrm{C}, 57.63 ; \mathrm{H}, 10.70 ; \mathrm{N}$, 8.19 ; $\mathrm{P}, 9.09$.

\section{Synthesis of diethyl}

\section{(1-phenyl-3-(phenylamino)pyrrolidin-2-yl)phosphonate 17f.}

Identical to procedure 17a except adding phenylamine and was obtained in $76 \%$ isolated yield.

${ }^{1} \mathrm{H}$ NMR (300 MHz, Chloroform d): ${ }^{1} \mathrm{H}$ NMR (300 MHz, Chloroform d): $\delta$ $1.22\left(\mathrm{dt}, 6 \mathrm{H}, J_{\mathrm{HH}}=6.9 \mathrm{~Hz},{ }^{3} J_{\mathrm{HP}}=2.1 \mathrm{~Hz}\right.$ ), $1.73-2.20$ (overlap, $3 \mathrm{H}$ ), $2.58-2.80$ (m, 2H), $3.00-3.10(\mathrm{~m}, 1 \mathrm{H}), 4.08-4.13(\mathrm{~m}, 4 \mathrm{H}), 7.21-7.35$ (overlap, 10$) ;{ }^{31} \mathrm{P}$ NMR (121.4 MHZ, Chloroform d): $\delta 30.98 ;{ }^{13} \mathrm{C}$ NMR $(75.5 \mathrm{MHz}$, Chloroform d): $16.2\left(\mathrm{~d},{ }^{3} J_{\mathrm{PC}}=6.8\right), 29.8,39.9,48.8\left(\mathrm{~d},{ }^{1} J_{\mathrm{PC}}=132.1 \mathrm{~Hz}\right), 47.0,61.0\left(\mathrm{~d},{ }^{2} J_{\mathrm{PC}}=2.8\right.$ 
$\mathrm{Hz}), 62.5\left(\mathrm{~d},{ }^{2} J_{\mathrm{PC}}=5.7 \mathrm{~Hz}\right), 127.9,128.8,129.2,141.0 ; \mathrm{MS}(\mathrm{EI}): \mathrm{m} / z(\%) 374(0.7)$, 373 (1.2), 297 (30.0), 255 (20.1), 220 (100), 189 (35.9), 137 (28.7), 89 (18.7), 77 (67.9), 65 (30.0); Anal. Calcd. for $\mathrm{C}_{20} \mathrm{H}_{27} \mathrm{~N}_{2} \mathrm{O}_{3} \mathrm{P}: \mathrm{C}, 64.16 ; \mathrm{H}, 7.27 ; \mathrm{N}, 7.48 ; \mathrm{P}, 8.27$. Found: C, 63.98; H, 7.13; N, 7.60; P, 8.41.

\section{Synthesis of diethyl}

\section{(1-heptyl-3-(heptylamino)pyrrolidin-2-yl)phosphonate $17 \mathrm{~g}$.}

Identical to procedure 17a except adding heptylamine and was obtained in $75 \%$ isolated yield.

${ }^{1} \mathrm{H}$ NMR (300 MHz, Chloroform d): $\delta 0.88\left(\mathrm{t}, 3 \mathrm{H}, J_{\mathrm{HH}}=5.7 \mathrm{~Hz}\right), 0.96(\mathrm{t}, 3 \mathrm{H}$, $\left.J_{\mathrm{HH}}=5.7 \mathrm{~Hz}\right), 1.28\left(\mathrm{dt}, 6 \mathrm{H}, J_{\mathrm{HH}}=7.2 \mathrm{~Hz},{ }^{3} J_{\mathrm{HP}}=2.1 \mathrm{~Hz}\right), 1.30-1.60$ (overlap, $16 \mathrm{H}), 1.74-2.18$ (overlap, 13H), $2.93-3.20(\mathrm{~m}, 1 \mathrm{H}), 4.04-4.10(\mathrm{~m}, 4 \mathrm{H}) ;{ }^{31} \mathrm{P}$ NMR (121.4 MHZ, Chloroform d): $\delta 32.25 ;{ }^{13} \mathrm{C}$ NMR (75.5 MHz, Chloroform d): 13.6, 14.0, $16.0\left(\mathrm{~d},{ }^{3} J_{\mathrm{PC}}=6.1\right), 22.2,22.7,26.9,27.0,27.9,28.2,28.8,29.0,29.4$, $29.8,31.0\left(\mathrm{~d},{ }^{1} J_{\mathrm{PC}}=135.8 \mathrm{~Hz}\right), 33.3,45.2,46.9\left(\mathrm{~d},{ }^{2} J_{\mathrm{PC}}=3.2 \mathrm{~Hz}\right), 49.0,53.0,60.8$ $\left(\mathrm{d},{ }^{2} J_{\mathrm{PC}}=8.6 \mathrm{~Hz}\right) ; \mathrm{MS}(\mathrm{EI}): \mathrm{m} / z(\%) 418$ (0.7), 417 (0.9), 412 (11.2), 396 (14.8), 345 (20.7), 289 (49.6), 235 (33.8), 189 (100), 135 (60.3), 87 (57.7), 43 (45.8); Anal. Calcd. for $\mathrm{C}_{22} \mathrm{H}_{47} \mathrm{~N}_{2} \mathrm{O}_{3} \mathrm{P}: \mathrm{C}, 63.12 ; \mathrm{H}, 11.32 ; \mathrm{N}, 6.69 ; \mathrm{P}, 7.40$. Found: C, 62.91; H, $11.17 ; \mathrm{N}, 6.81 ; \mathrm{P}, 7.56$.

\section{Synthesis of diethyl}

(1-phenethyl-3-(phenethylamino)pyrrolidin-2-yl)phosphonate17h.

Identical to procedure 17a except adding phenylethylamine and was obtained in $79 \%$ isolated yield.

${ }^{1} \mathrm{H}$ NMR (300 MHz, Chloroform d): ${ }^{1} \mathrm{H}$ NMR (300 MHz, Chloroform d): $\delta$ $1.24\left(\mathrm{dt}, 6 \mathrm{H}, J_{\mathrm{HH}}=6.9 \mathrm{~Hz},{ }^{3} J_{\mathrm{HP}}=1.67 \mathrm{~Hz}\right), 1.58-2.18$ (overlap, $3 \mathrm{H}$ ), $2.14-2.85$ (overlap, 7H), $4.04-4.15$ (m, 4H), 7.17 - 7.31 (overlap, 10); ${ }^{31} \mathrm{P}$ NMR (121.4 MHZ, Chloroform d): $\delta 31.78 ;{ }^{13} \mathrm{C}$ NMR (75.5 MHz, Chloroform d): $16.2\left(\mathrm{~d},{ }^{3} J_{\mathrm{PC}}\right.$ = 7.2), 31.3, 32.8, 41.5, $49.5\left(\mathrm{~d},{ }^{1} \mathrm{~J}_{\mathrm{PC}}=132.9 \mathrm{~Hz}\right), 48.2,55.6,57.9,61.0\left(\mathrm{~d},{ }^{2} J_{\mathrm{PC}}=4.2\right.$ $\mathrm{Hz}), 60.9\left(\mathrm{~d},{ }^{2} J_{\mathrm{PC}}=5.7 \mathrm{~Hz}\right), 127.7,127.0,128.2,141.5 ; \mathrm{MS}(\mathrm{EI}): \mathrm{m} / z(\%) 430(0.7)$, 429 (0.8), 353 (18.9), 325 (32.1), 276 (100), 234 (22.8), 188 (45.9), 135 (57.7), 105 (77.8), 77 (46.8), 69 (66.7); Anal. Calcd. for $\mathrm{C}_{24} \mathrm{H}_{35} \mathrm{~N}_{2} \mathrm{O}_{3} \mathrm{P}: \mathrm{C}, 66.96 ; \mathrm{H}, 8.19 ; \mathrm{N}$, 6.51; P, 7.19. Found: C, 67.08; H, 8.31; N, 6.37; P, 7.06.

\section{Acknowledgements}

This research was supported by Al Quds University funding (AAQ, HD, AJ). The work (GTA) was performed in the framework of the Program for Basic Research of State Academies of Sciences for 2013-2020, also this work (DVM) was supported by the Russian Science Foundation (Grant No. 18-73-10030).

\section{Conflicts of Interest}

The authors declare no conflicts of interest regarding the publication of this paper.

\section{References}

[1] Gabay, J.E. (1994) Ubiquitous Natural Antibiotics. Science, 264, 373. 
https://doi.org/10.1126/science.8153623

[2] Walsh, C. (2003) Antibiotics: Actions, Origins, Resistance. American Society for Microbiology, Washington DC, 1-335. https://doi.org/10.1128/9781555817886

[3] Luzhetsky, A., Pelzer, S. and Bechthold, A. (2007) The Future of Natural Products as a Source of New Antibiotics. Expert Opinion on Investigational Drugs, 8, 608-613.

[4] Grenni, P., Ancona, V. and Caracciolo, A.B. (2018) Ecological Effects of Antibiotics on Natural Ecosystems: A Review. Microchemical Journal, 136, 25-39. https://doi.org/10.1016/j.microc.2017.02.006

[5] Engel, R. (1977) Phosphonates as Analogues of Natural Phosphates. Chemical Reviews, 77, 349-367. https://doi.org/10.1021/cr60307a003

[6] Romanenko, V.D. and Kukhar, V.P. (2006) Fluorinated Phosphonates: Synthesis and Biomedical Application. Chemical Reviews, 106, 3868-3935. https://doi.org/10.1021/cr051000q

[7] Hecker, S.J. and Erion, M.D. (2008) Prodrugs of Phosphates and Phosphonates. Journal of Medicinal Chemistry, 518, 2328-2345. https://doi.org/10.1021/jm701260b

[8] Satani, N., Lin, Y.-H., Hammoudi, N., Raghavan, S., Georgiou, D.K. and Muller, F.L. (2016) ENOblock Does Not Inhibit the Activity of the Glycolytic Enzyme Enolase. PLoS ONE, 11, e0168739. https://doi.org/10.1371/journal.pone.0168739

[9] Dembitsky, V.M., Gloriozova, T.A. and Savidov, N. (2018) Steroid Phosphate Esters and Phosphonosteroids and Their Biological Activities. Applied Microbiology and Biotechnology, 102, 7679-7692. https://doi.org/10.1007/s00253-018-9206-Z

[10] Dembitsky, V.M., Al Quntar, A.A.A., Haj-Yehiaa, A. and Srebnik, M. (2005) Recent Synthesis and Transformation of Vinylphosphonates. Mini-Reviews in Organic Chemistry, 2, 91-109. https://doi.org/10.2174/1570193052774090

[11] Al Quntar, A.A.A., Dembitsky, V.M. and Srebnik, M. (2008) $\alpha$-, $\beta$-, $\gamma$ - and $\omega$-Cyclopropylphosphonates. Preparation and Biological Activity. Organic Preparations and Procedures International, 40, 505-542.

https://doi.org/10.1080/00304940809458117

[12] Al Quntar, A.A.A., Srebnik, M., Terent'ev, A.O. and Dembitsky V.M. (2014) Cyclobutyl- and Cyclobutenylphosphonates: Synthesis, Transformationsand Biological Activities. Mini-Reviews in Organic Chemistry, 11, 445-461. https://doi.org/10.2174/1570193X1104140926170132

[13] Haji, M. (2016) Multicomponent Reactions: A Simple and Efficient Route to Heterocyclic Phosphonates. Beilstein Journal of Organic Chemistry, 12, 1269-1301. https://doi.org/10.3762/bjoc.12.121

[14] Ohba, K., Watabe, H., Yoshida, J., Shomura, T., Sezaki, M. and Ishikawa, T. (1985) Synthesis of 4-Phosphono B-Lactams and Related Azaheterocyclic Phosphonates. Jpn. Patent, 60,224,493 (Chem. Abstr., 1986, 104, 107918y).

[15] Ohba, K., Sato, Y., Sasaki, T. and Sezaki, M. (1986) Studies on a New Phosphonic Acid Antibiotic, SF-2312. Scientific Reports of Meiji Seika Kaisha, 25, 18-23.

[16] Fields, S.C. (1999) Synthesis of Natural Products Containing a C-P Bond. Tetrahedron, 55, 12237-12273. https://doi.org/10.1016/S0040-4020(99)00701-2

[17] Leonard, P.G., Satani, N., Maxwell, D., Lin, Y.H., Hammoudi, N., Peng, Z. and Pisaneschi, F. (2016) SF2312 Is a Natural Phosphonate Inhibitor of Enolase. Nature Chemical Biology, 12, 1053-1058. https://doi.org/10.1038/nchembio.2195

[18] Pisaneschi, F., Lin, Y.H., Leonard, P.G., Satani, N., Yan, V.C., Hammoudi, N., Raghavan, S., Todd, M., Link, K., Georgiou, D., Czako, B. and Muller, F. (2019) The 3S Enantiomer Drives Enolase Inhibitory Activity in SF2312 and Its Analogues. Mole- 
cules, 24, 2510-2516. https://doi.org/10.3390/molecules24132510

[19] Owotoki, W., Geffken, D. and Kurz, T. (2006) Synthesis of 1-Hydroxypyrrolidin-2, 5-Dione Derivatives of the Phosphonic-Hydroxamic Acid Antibiotic SF-2312. Australian Journal of Chemistry, 59, 283-288. https://doi.org/10.1071/CH06058

[20] Owotoki, W. (2007) Structure-Activity-Studies on the Natural Antibacterial Compound SF-2312. PhD Thesis Dissertation, Hamburg.

[21] Hanaya, T. and Itoh, C. (2010) An Efficient Synthesis of Antibiotic SF-2312 (3-Dihydroxyphosphoryl-1,5-dihydroxy-2-pyrrolidone). Heterocycles, 82, 1675-1683. https://doi.org/10.3987/COM-10-S(E)80

[22] Sadym, A., Lagunin, A., Filimonov, D. and Poroikov, V. (2003) Prediction of Biological Activity Spectra via the Internet. SAR and QSAR in Environmental Research, 14, 339-347. https://doi.org/10.1080/10629360310001623935

[23] Toccaceli, P., Nouretdinov, I. and Gammerman, A. (2017) Conformal Prediction of Biological Activity of Chemical Compounds. Annals of Mathematics and Artificial Intelligence, 81, 105-123. https://doi.org/10.1007/s10472-017-9556-8

[24] Lagunin, A.A., Goel, R.K., Gawande, D.Y., Priynka, P., Gloriozova, T.A. and Dmitriev, A.V. (2014) Chemo- and Bioinformatics Resources for in Silico Drug Discovery from Medicinal Plants beyond Their Traditional Use: A Critical Review. Natural Product Reports, 31, 1585-1611. https://doi.org/10.1039/C4NP00068D

[25] Poroikov, V.V., Filimonov, D.A., Gloriozova, T.A., Lagunin, A.A., Druzhilovskiy, D.S. and Rudik, A.V. (2019) Computer-Aided Prediction of Biological Activity Spectra for Chemical Compounds: Opportunities and Limitations. Russian Chemical Bulletin, 68, 2143-2154. https://doi.org/10.1007/s11172-019-2683-0

[26] Filimonov, D.A., Lagunin, A.A., Gloriozova, T.A., Rudik, A.V., Druzhilovskii, D.S., Pogodin, P.V. and Poroikov, V.V. (2014) Prediction of the Biological Activity Spectra of Organic Compounds Using the PASS Online Web Resource. Chemistry of Heterocyclic Compounds, 50, 444-457. https://doi.org/10.1007/s10593-014-1496-1

[27] Vil, V.A., Gloriozova, T.A., Poroikov, V.V., Terent'ev, A.O., Savidov, N. and Dembitsky, V.M. (2019) Peroxy Steroids Derived from Plant and Fungi and Their Biological Activities. Applied Microbiology and Biotechnology, 102, 7657-7667. https://doi.org/10.1007/s00253-018-9211-2

[28] Dembitsky, V.M., Savidov, N., Poroikov, V.V., Gloriozova, T.A. and Imbs, A.B. (2018) Naturally Occurring Aromatic Steroids and Their Biological Activities. Applied Microbiology and Biotechnology, 102, 4663-4674. https://doi.org/10.1007/s00253-018-8968-7

[29] Van Assche, I., Soroka, M., Haemers, A., Hooper, M., Blanot, D. and Heijenoort, J. (1991) Synthesis and Antibacterial Evaluation of Phosphonic Acid Analogues of Diaminopimelic Acid. European Journal of Medicinal Chemistry, 26, 505-515. https://doi.org/10.1016/0223-5234(91)90146-E

[30] Frejaville, C., Karoui, H., Tuccio, B., Le Moigne, F., Culcasi, M., Pietri, S., Lauricella, R. and Tordo, P. (1994) 5-Diethoxyphosphoryl-5-methyl-1-pyrroline N-oxide (DEPMPO): A New Phosphorylated Nitrone for the Efficient in Vitro and in Vivo Spin Trapping of Oxygen-Centred Radicals. Journal of the Chemical Society, Chemical Communications, No. 15, 1793-1794. https://doi.org/10.1039/c39940001793

[31] Pietri, S., Miollan, M., Martel, S., Le Moigne, F., Blaive, B. and Culcasi, M. (2000) A New Class of Low Toxic Highly Sensitive 31P NMR pH Indicators. Journal of Biological Chemistry, 275, 19505-19512.

[32] Robiette, R. and Marchand-Brynaert, J. (2001) $\alpha$ - and $\beta$-Phosphorylated Amines 
and Pyrrolidines, a New Class of Low Toxic Highly Sensitive 31P NMR pH Indicators. Journal of the Chemical Society, Perkin Transactions, 11, 2155-2158.

[33] Kaboudin, B., Karami, L., Kato, J.-Y., Aoyama, H. and Yokomatsu, T. (2013) A Catalyst-Free, Three-Component Decarboxylative Coupling of Amino Acids with Aldehydes and H-Dialkylphosphites for the Synthesis of $\alpha$-Aminophosphonates. Tetrahedron Letters, 54, 4872-4875. https://doi.org/10.1016/j.tetlet.2013.06.129

[34] Ohba, K. and Dewik, H. (2016) Synthesis of Substituted Pyrrolidinylmethylphosphonates by Addition of Amines to 5-Chloro-1-pentenylphosphonate. Tetrahedron Letters, 57, 189-191. https://doi.org/10.1016/j.tetlet.2015.11.097

[35] Al Quntar, A.A.A., Dweik, H. and Dembitsky, V. (2020) Facile Synthesis of 2-Amino-cyclobutenylphosphonates and Their Predicted Biological Activity. Russian Journal of Organic Chemistry, 56, 139-142.

https://doi.org/10.1134/S1070428020010212 